\title{
Applications of TestWizard in the Fibre Channel Test System
}

\author{
Xiucai Zhao \\ Science and Technology on Electronic Test \& \\ Measurement Laboratory \\ Qingdao, China \\ Zhaoxiucai1973@163.com
}

\author{
Panwei Wang \\ Science and Technology on Electronic Test \& \\ Measurement Laboratory \\ Qingdao, China \\ eiqd@ei41.com
}

\begin{abstract}
As more and more Fiber Channel related products are being used, the needs of a FC test $\&$ analysis system are more urgent now. A FC system is a test \& analysis system that can test the performance of the network, can capture the Fiber Channel data and can simulate and analysis the protocols. This paper focuses on the TestWizard test software's application for a particular model of FC test \& analysis system. Based on the cadre and configuration tools provided by TestWizard, the present FC system has many advances: a friendly user interface, a customizable test application, a tidy data synthesis management, reusable and portable TPs. The application of the FC system has greatly improved the efficiency of FC end devices' developing, debugging, interlinking, testing and verifying. The present FC test $\&$ analysis system achieves the expected results either.
\end{abstract}

Keywords- Fibre Channel; TestWizard; test program; Component; protocol analysis; configuration; secondary development; data management; reusable

\section{INTRODUCTION}

With the rapid development and wide application of computer technology and communication technology, complex electronic devices are developing quickly toward modularization, integration and open system frameworks; Data transferred within systems increases rapidly. This results in the need for a high-bandwidth, low latency, good scalability and reliable transmission transfer network. FC(Fiber Channel) is such a high-performance and mutual network technology that can meet those needs.

Currently, Fibre Channel is used widely not only in the avionics system; with the developing of data centers, it is also used in the Storage Area Network (SAN). Thus, Fibre Channel's protocol Simulation, Real-time monitoring, data logging, protocol analysis, error injection and so on, are becoming more and more important; Especially in FC end devices' developing and debugging, in testing and verifying the communications between FC nodes and/or FC switches, even in evaluating the whole FC network's performance.

Therefore, it is especially significant to develop a set of these functions in one FC test and analysis system that used to improve the efficiency of FC end devices' developing, debugging, interlinking, testing and verifying.

TestWizard is an application developed by CETC41; it has such features as test development, test execution and test management. The software not only provides easy-touse basic functions and a friendly man-machine interface for the operators of ATS, but also provides a flexible and practical framework for the development managers of ATS. It can significantly improve the efficiency of test application development, test task execution and test data management. In this paper, we will discuss the application of TestWizard software in one FC test \&analysis system.

\section{BASIC TESTING REQUIREMENTS}

Fibre Channel is an open high-speed serial data communication standard created by the American National Standards Institute (ANSI) X3T9.3 Working Group (now X3T11 Working Group); it guidelines the communication between computers or computer and I/O devices.

Since 1988, with the supporting of FCA and FCLG, the standard has been promoted, carried out and implemented; a great variety of FC related products has been developed too. Fibre Channel has lots of advantages, such as highbandwidth, low latency, non-distance sensitive, flexible topology, various upper-layer protocols support, and so on; thus, it has not only been widely adopted in commercial areas, it has also becoming the preferred standard for airborne avionics unified network.

Currently, Domestic FC studies are focused on the application of the system and the software development. The core technologies such as FC protocol control chips and IP cores are in its infancy. FC switches, FC host adapters and FC related boards have to be imported from foreign manufacturers; The cost of importing those products are expensive, the procurement and maintenance are difficult, and it is not conducive to long-term sustainable development too.

Therefore, using FC simulation test tools to research into FC related products and technologies is significant to our national defense construction and national economic development; it can also help to research, innovate, use and maintain our own independent-intellectual-property-rights FC products and related application platforms.

From the point of the avionics interconnection system design and use, the most important technical specifications of network performance testing are the network throughput and the end-to-end message time delay characteristics, thus it is very important to test the average throughput and the average time delay of the FC network.

In addition, Along with the research, production and experimental verification of FC-related products, it not only 
need to simulate the FC terminal equipment to the target simulation, product testing and conformance testing, but also need to collect and record some or all of the data on the Fibre Channel bus For the FC protocol analysis and network performance evaluation.

Thus, The FC test and analysis system (hereinafter referred to as the system) not only need to perform the FC network performance testing, the data simulating, bus data collecting and protocol analyzing, but also need to be provided with some secondary development capacity that allows the technicians to utilize the existing hardware and software resources to expanding the testing capabilities; it forms a flexible evaluation development environment to support Fibre Channel simulation testing, evaluation data collection and test program development; finally, the system build a solid data base for the follow-up performance assessment of the whole FC system.

\section{FC TEST AND ANALYSIS SYSTEM COMPOSITION}

The entire system is connected to the measured object FC network through the FC interface, as illustrated in Figure 1.The system consists of hardware platform and software platform; hardware platform includes a master control device, data storage devices, FC switches, laser printers, and test cables. The software platform includes an operating system, test support software, test management software and a series of test applications.

The master control device is the core device of the system, it includes a built-in FC communication interface card, FC simulation test card and Ethernet interface card; the master control device performs such functions: FC network performance test, data simulation, bus data collection and protocol analysis and assessment. The data storage device is made up of a memory module a FC controller and some enterprise-class hard disks, those devices are connected to the system by the FC host. The data storage device functions as the massive storage device for bus data. FC switch is used to connect the measured objects to the system; it can perform a full board-level speed seamless connection between the system and the measured FC network. Laser printer is used for the printout of test report. The system software is running under the Chinese Edition of Microsoft's Windows XP operating system. The test support software includes the Oracle10g database system, Microsoft Office2003, integrated development environment NI LabWindows/CVI8.5. The test management software is CETC41's TestWizard V1.0. The data storage device is connected to the FC communication interface card by the optical fiber. The FC test simulator card embedded in the master control device provides a $1 / 2 / 4 \mathrm{Gbps}$ dual-port full board-level speed data generating/simulating and monitoring/analyzing capabilities. You can connect the simulator card directly to the measured FC networks, or you can use a FC switch to format a FC Link then connect it to the measured FC networks. The system master control device configures the data storage devices and the FC switch via the LAN.

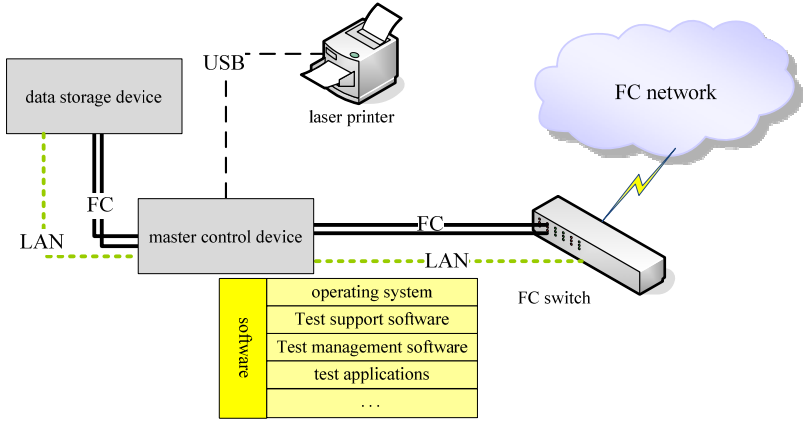

Figure 1. General view of the system

\section{THE SPECIFIC APPLICATION OF TESTWIZARD}

The TestWizard software provides a fundamental tool for users to customizing the user interface, test task execution, data analysis, and report forms generation. It is particularly suitable for advanced users to do the test design verification, functional performance verification as well as the test system customizing. Using the framework provided by TestWizard, technical staffs can easily integrate a variety of hardware and software test resources, and focus on the development of test programs, accelerate the development of the test application and test program's multiplexing, and improve the functional performance; thus develop and maintenance the test application quickly and better.

In the specific FC Test and analysis system, we use TestWizard as the test management software to develop, execute and manage test programs. According to test resources and test system requirements, we configure the operating platform's interface, customizing the test applications, comprehensive managing of the bus test data, ect. They will be described in the following.

\section{A. Configuration of the platform interface}

In the FC test analysis system, the operating platform is a uniform interface for human-computer interaction, interactions between users and the system test resources are processed by the operating platform. The platform is a graphical and easy-to use user interface. Using the maintenance tools provided by the TestWizard software one can easily customize and configure the operating platform. Configuration window is shown in Figure 2.

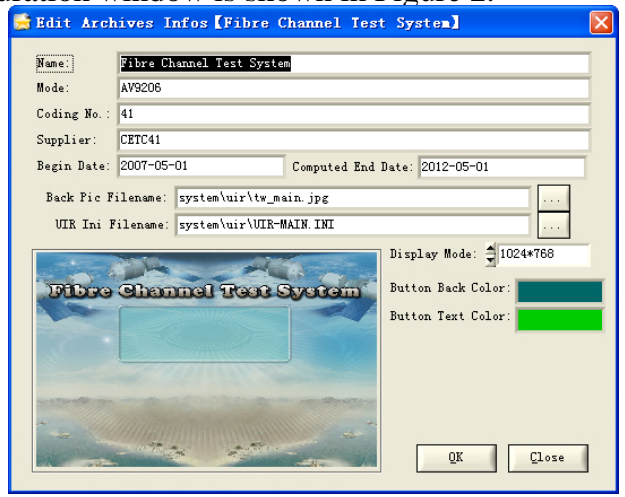

Figure 2. Configuration Archives window 
As illustrated in figure 2, the name, model, supplier and etc. can be edited directly. The background image of the main interface for the operating platform can be JPG, PCX, and BMP ... file formats, the pixel sizes can be $640 \times 480$, $800 \times 600$ or $1024 \times 768$. The Configuration Interface file is .INI format, it can be edited using the text editing tool [UltraEdit-32] embedded in TestWizard, items be configured are: the number of control buttons, each button's property information as well as the tips ..., as illustrated in Figure 3.

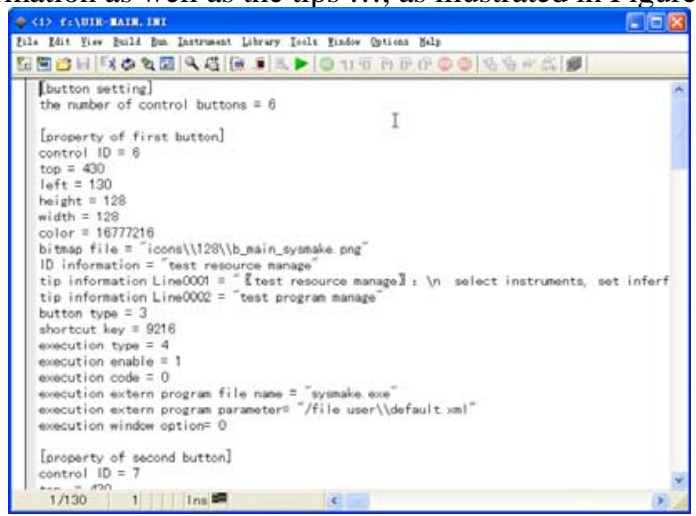

Figure 3. File configuration window

After the user completes customizing the interface, one can see the effect of the customized interface on the operating platform. As illustrated in figure 4, that is a successfully customized operating platform interface. Using that interface, the user can visually study the main functions, and do the test processing quickly and easily.

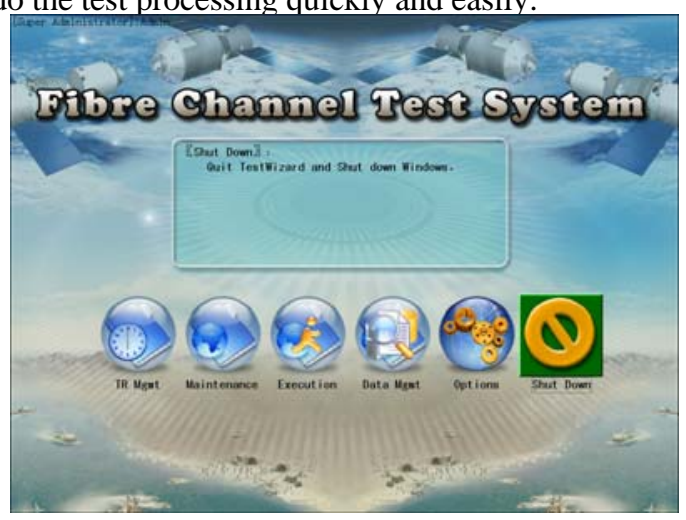

Figure 4. Platform interface diagram

\section{B. Customization and programing of test procedures}

The environment of the test program can affect the design method of the test procedure and the final accomplished results. Nowadays, the mainstream development methods of test programs include procedural programming, graphical programming and the functional components assembling method. The procedural programming method requires users to be familiar with the programming languages (such as $\mathrm{C}$ language), the graphical programming method is visual and easy to use, but it's low programing efficiency is a great disadvantages for large and complex software development. In comparison, the functional components assembling method takes advantages of the existing functional components to reform a new functioning as required, it is visual, simple and quick. Thus, TestWizard provides a full solution in the assembly of component-based test program development. It is greatly facilitate in the secondary development of test programs for users.

In the FC test analysis system, in order to improve the efficiency of the secondary development for users, several parameter components are published specially. Those components include throughput test, data frames transmitting and data frames statistics. So, users can directly use these parameter components when they do the secondary development in TestWizard. Generally, it requires the following steps to customize test programs in TestWizard.

First, Requirement analyze of the test program: after completing the measurability analyzing of the tested object and asserting of the test tasks, one can do the modularizing work of the test tasks. In TestWizard, in order to facilitate management, a test mission is in principle corresponds to a test program. And a test program is designed as a test sequence, which consists of one or more relatively independent functional modules (i.e. components). Taking interactive bus data recording as an example, first, setup the capture parameters in FC simulation test board panel, then, start the 'data frame transmitting component' to transmit data on the FC communication link, finally guide users to archive information step by step. In this process, there at least needs to be divided into three functional modules: parameter capturing setups, data frame transmission, and test information archiving. Tips and execution conformation are set in according to user's habits.

Second, the design and implementation of test program: using existing components as the test steps for the sequence assembly, or using revising and improving the existing test sequences as the test steps. In TestWizard, the user can use seven types of components for the sequence assembly; those components are: test parameters component, executable program component, multimedia tips component, popup style information component, virtual instruments component, delay control component and information archiving component. The interfaces of those component models are open to the public, users can develop the customized components by the mature third-party development tools (such as CVI). Taking the frequently used test parameter component as an example, TestWizard provides a management tool for test parameters component library, as illustrated in Figure 5. The user can easily add, delete, edit and debug test parameters component via the tool. In the FC test analysis system, we develop several specific parameter components for the special needs of test tasks, which are throughput test, the data frame statistics and data frames transmission. By using those parameter components, we develop programs for performance test and functional verification of FC nodes, FC switches and FC networks. 


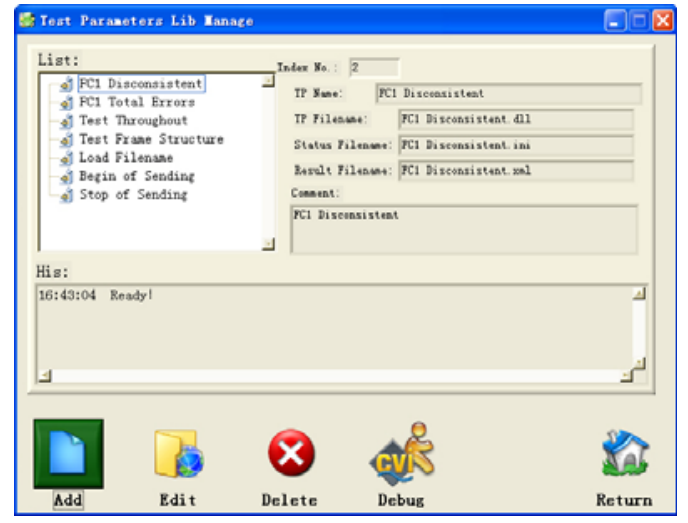

Figure 5. Test parameters component management tool

Third, test programs' debugging: after loading the test program through the test program maintenance wizard, there enters the core module of TestWizard: test program maintenance software, as shown in Figure 6. By using this development tool, users can debug, modify or validate the test sequences.

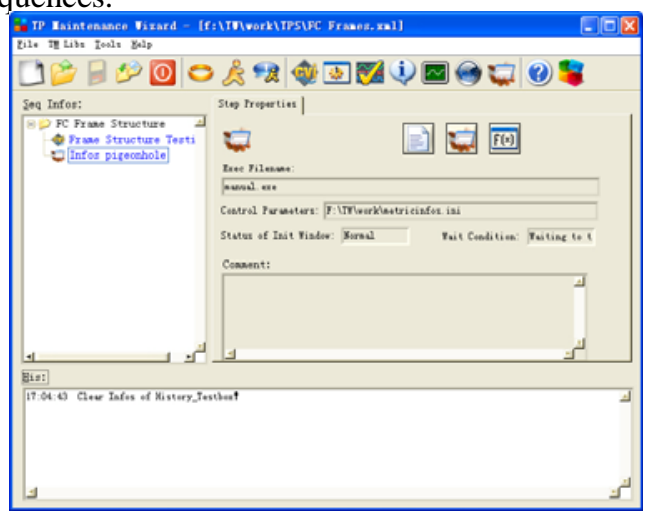

Figure 6. Test program maintenance software’s user interface

The information of test programs will be stored in XML format (See Figure 7 below) after they are debugged successfully. Thus, programs developed in this way are powerful in reusability and easy in modification and maintenance. The advanced developers can complete the test procedures customized development by using a common text edit tool.

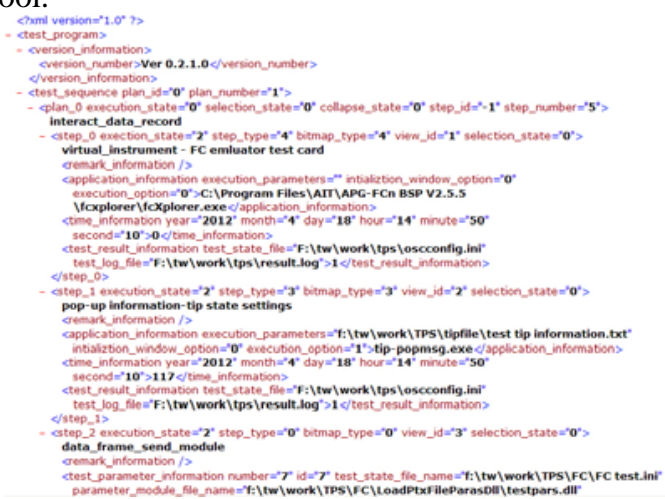

Figure 7. information stored in XML format

\section{Test data management}

In the FC test and analysis system, users need to use the test programs to control hardware resources embedded in the system. For a topological link formed for the measured object, under the premise that it does not affect the structure and characteristics of the measured object information transmission, it gets the information from the FC Bus, and ensures that all data are recorded and analyzed. Bus throughput, the memory interface speed and storage space size are not the only things to be considered, the user's habits need to be considered either when design a specific tool for test information querying, archiving, browsing or export analyzing.

In TestWizard, the data management software provides such functions: test information querying and exploring, quering results outputting, test information deleting and information archiving, etc. The user interface is shown in Figure 8. The report formats can be XML, XLS (Excel spreadsheet table format) and so on, thus do a greatly convenience for users.

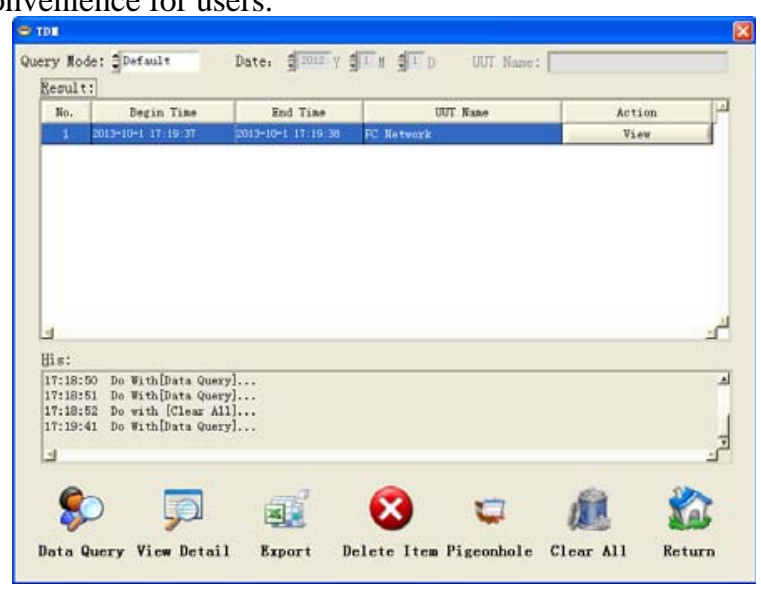

Figure 8. Data management software user interface

In the FC test analysis system, bus data collected are massive, sampling hundreds of MB or even GB per time is common, therefore the system has the Oracle database tool embedded. The system supplies specialized data storage archive component and test information browse component for data management. At the same time, in order to improve the processing efficiency of data storage and transmission, in TestWizard, storage archive component and information browse component are integrated with WinRAR, which is powerful and compression rate is high. A cursory statistics: a 300MB-size data frame capturing file can be compressed and stored in less than 15 seconds, the compressed file size is less than $6 \mathrm{MB}$, the compression rate is great than $98 \%$; the results is desirable. Information browse is also fast and convenient. Detailed information exploring results are shown in Figure 9, after lossless test data exportation, the user can use a professional tool to analyze or evaluate the protocols. 


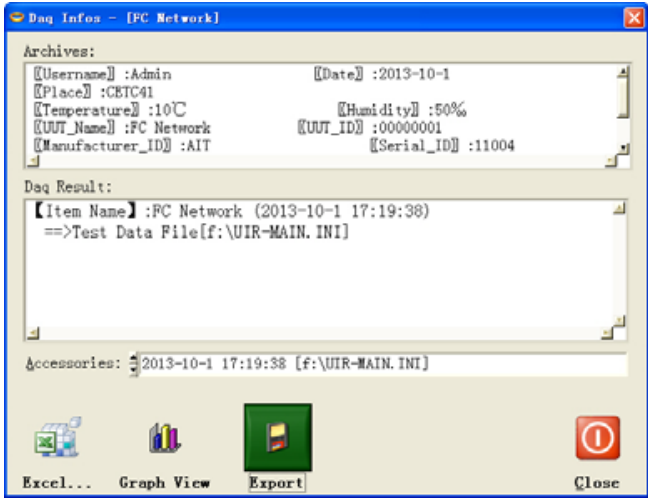

Figure 9. Test results exportation

\section{CONCLUSION}

In the specific model FC test and analysis system, we successfully developed several test components/functional modulars for users' particular applications. Using the infrastructure and configuration tools provided by TestWizad, it is simple and convenient to configure the user interface, customize test applications and manage application data, the test applications developed are reusable and portable too.
The trial of the FC test and analysis system is successful; it has not only successfully completed the performance testing of the optical channel link (throughput, delay, etc.), capture and storage of data frames, protocol analysis of data frames, and so on; it has also simulated the end-to-end test verification of the FC terminal devices, including data generation, data transmission, data corruption, error injection, and error detection.

The application of the FC system has greatly improved the efficiency of FC terminal devices' developing, debugging, interlinking, testing and verifying. The successful application of TestWizard shows that, the specific FC test \& analysis system achieves the expected results: high degree of modularity, fully functional test capabilities, powerful abilities of secondary development and friendly user interface. We hope that the methods and contents discussed in this paper may provide some help for similar test applications.

\section{REFERENCES}

[1] Robert W.Kembel Fibre Channel:A Comprehensive Introduction. Northwest Learning Associates,Inc. 2002,pp.1-37.

[2] LabWindows/CVI Programmer Reference Manual. National Instuments,1998 pp.186-207. 